\title{
Presión arterial en embarazos normales de la Ciudad de México
}

\section{Blood pressure in normal pregnancies from Mexico City}

\author{
Héctor Oviedo-Cruz ${ }^{1 *}$ y Marco A. Cortés-Martínez ${ }^{2}$ \\ ${ }^{1}$ Departamento de Medicina Materno-Fetal, Centro Médico para la Atención Fetal Especializada; ${ }^{2}$ Departamento de Bioquímica y Calidad, \\ Laboratorio CEMAFE S.A. de C.V., Ciudad de México, México
}

\begin{abstract}
Resumen
Objetivo: Determinar la presión arterial (PA) normal en embarazadas en la Ciudad de México. Método: Estudio transversal descriptivo de la PA en embarazos normales de feto único en la Ciudad de México. Las mediciones siguieron un método estandarizado con uso de dispositivos electrónicos automatizados. Se construyeron valores de referencia de las presiones sistólica (PS), diastólica (PD) y arterial media (PAM). Se probaron los efectos de las características maternas y de la gestación sobre la PA. Los intervalos de referencia de la PA se ajustaron para las variables significativas. Resultados: En 1,056 mujeres con edad media de 33 años y $160 \mathrm{~cm}$ medios de estatura se realizaron 1,915 mediciones entre las 5 y 41 semanas de edad gestacional (EG) con peso medio de $65 \mathrm{~kg}$. La PA durante el embarazo fue de 102.7/67.2 \pm 9.3/7.4 DE mmHg y 79.0 \pm 7.4 DE mmHg la PAM. La PA tuvo una relación cuadrática con la EG, la más baja en el segundo trimestre. La PS y la PD mostraron una correlación lineal $(r=0.71)$. El peso materno tuvo el efecto más significativo sobre PS y PAM; la estatura sobre la PD. La edad, etnia, paridad, consumo de tabaco y antecedente familiar ejercieron efectos diferentes en cada PA. La gestación no tuvo efecto significativo en el modelo multivariado de la PS. Conclusiones: La PA se halló por debajo de la norma convencional en embarazadas. Los criterios diagnósticos para hipertensión en el embarazo deben revisarse; los valores de referencia pueden ajustarse a características maternas y gestacionales.
\end{abstract}

Palabras clave: Presión arterial. Embarazo. Valores de referencia. Preeclampsia. México.

\begin{abstract}
Objective: To determine the normal blood pressure (BP) in pregnancy, Mexico City. Methods: A cross-sectional observational study was carried out of BP on singleton normal pregnancies in Mexico City. Measurements followed a standardised methodology using automated electronic devices. Reference values of systolic (SBP), diastolic (DBP) and mean arterial pressure (MAP) were constructed. Maternal and gestation characteristics effects on BP were tested. The reference ranges of BP were adjusted for significant variables. Results: On 1,056 women of 33 years old mean age and $160.0 \mathrm{~cm}$ mean height, 1,915 measurements were made between 5 and 41 weeks of gestational age (GA) with $65.0 \mathrm{~kg}$ of mean weight. The median BP throughout pregnancy was 102.7/67.2 \pm 9.3/7.4 SD mmHg, and $79.0 \pm 7.4 S D \mathrm{mmHg}$ the MAP. BP had a quadratic relationship with GA, being the lowest in $2^{\text {nd }}$ trimester. SBP and DBP had a $r=0.71$ linear correlation. Maternal weight had the most significant effect on SBP and MAP; height, on DBP. Maternal age, ethnic origin, parity, tobacco habit and family history had differential effects on BP. Gestation had no significant effect on SBP multivariate model. Conclusions: BP resulted lower than conventional standard for pregnant women. Diagnostic criteria for hypertension in pregnancy must be revised; reference values can be adjusted by maternal and gestation characteristics.
\end{abstract}

Key words: Blood pressure. Pregnancy. Reference values. Pre-eclampsia. Mexico.

\footnotetext{
Correspondencia:

Fecha de recepción: 08-05-2020

*Héctor Oviedo-Cruz

E-mail: HectorOC@ cemafe.org.mx

Fecha de aceptación: 03-09-2020

DOI: 10.24875/ACM.20000206

Disponible en internet: 02-07-2021 Arch Cardiol Mex. 2021;91(3):289-298 www.archivoscardiologia.com

1405-9940 / @ 2020 Instituto Nacional de Cardiología Ignacio Chávez. Publicado por Permanyer. Este es un artículo open access bajo la licencia CC BY-NC-ND (http://creativecommons.org/licenses/by-nc-nd/4.0/).
} 


\section{Introducción}

La hipertensión en el embarazo se define como una presión sistólica (PS) $\geq 140 \mathrm{mmHg}$, una presión diastólica (PD) $\geq 90 \mathrm{mmHg}$ o ambas en dos ocasiones, al menos con cuatro horas de diferencia ${ }^{1-4}$. Ésta ha sido la norma de cuidado por muchos años ${ }^{5}$. En la población no gestante, el American College of Cardiology (ACC) ha cambiado en fecha reciente la definición misma de hipertensión y respaldado umbrales más bajos con el objetivo de reducir la morbilidad cardiovascular a largo plazo ${ }^{6}$. La aplicación de estos intervalos más bajos en la población embarazada ha posibilitado una mejor detección de resultados adversos maternos y perinatales ${ }^{7,8}$.

Por otro lado, sólo hasta ahora se han tenido en cuenta los cambios fisiológicos de la presión arterial durante la gestación ${ }^{9}$ para construir intervalos de referencia para la valoración de mujeres embarazadas ${ }^{10}$. Sin embargo, los umbrales dependientes de la edad gestacional no se consideran en las guías de práctica clínica ${ }^{11}$.

Además, un estudio describió los efectos diferenciales de las características maternas y el historial médico sobre la presión arterial media (PAM) en los tres trimestres del embarazo ${ }^{12}$.

El objetivo de este estudio fue construir valores de referencia de la presión arterial durante la gestación, ajustados por características maternas específicas de la población.

\section{Materiales y métodos}

\section{Población de estudio}

Se realizó un estudio transversal en embarazos de feto único, vivo, intrauterino, de concepción espontánea y normal al momento de la ecografía fetal regular en un centro médico y laboratorio de medicina maternofetal de la Ciudad de México; la inclusión fue consecutiva de las embarazadas que concedieron su consentimiento para participar. Se incluyó a mujeres sanas de cualquier origen étnico; no se incluyó a pacientes con hipertensión crónica, diabetes, lupus eritematoso sistémico, síndrome antifosfolípidos ni otras enfermedades, incluso si no representaban un riesgo cardiovascular. Se excluyó a los embarazos con anomalías fetales cromosómicas, estructurales o del crecimiento intrauterino. No se incluyó a embarazos de fetos múltiples porque se han documentado diferencias en ellos para la presión arterial ${ }^{13}$ y la función cardíaca ${ }^{14}$; esto es motivo de otra investigación.
El estudio obtuvo la aprobación de los comités científico y bioética de dicho centro, con apego a la Declaración de Helsinki de la Asociación Médica Mundial para las investigaciones médicas en seres humanos.

\section{Características de las pacientes}

Las características clínicas se obtuvieron por cuestionario autoadministrado, verificadas por interrogatorio médico, e incluyeron edad materna, origen étnico (caucásico/hispano, afrocaribeño, sur asiático, este asiático u otro/mixto), hábito de fumar durante el embarazo (fumadora, nunca o abandono), antecedentes familiares de preeclampsia en la madre o la hermana de la paciente (sí o no) y antecedentes obstétricos que incluyan paridad (paridad o nuliparidad si no había embarazos previos de $\geq 24$ semanas de gestación) y embarazo previo con preeclampsia (sí o no); estas definiciones operativas son las que emplea la Fundación de Medicina Fetal' ${ }^{15}$ (nótese que la categoría "nulípara" no es igual a "primer embarazo"; los abortos y nacimientos $<24$ semanas no tienen interés operacional).

El peso y la estatura maternos se obtuvieron con báscula y estadímetro clínicos convencionales analógicos calibrados. La edad de la gestación se estableció por ecografía o por fecha de última menstruación corroborada por ecografía.

\section{Método estandarizado para medir la presión arterial en el embarazo}

De acuerdo con las recomendaciones internacionales ${ }^{11}$ desarrolladas por la Fundación de Medicina Fetal ${ }^{12,16-18}$ y adoptadas en fecha reciente por la Federación Internacional de Ginecología y Obstetricia ${ }^{19}$, se especifica el uso de dispositivos electrónicos automatizados para evitar sesgo humano y de intercambiabilidad por auscultación ${ }^{20}$. En vez de establecer un número fijo de lecturas, se toman las necesarias para alcanzar el estado basal de la embarazada; en un estudio se notificó que hay casos que requieren cuatro 0 más lecturas ${ }^{18}$; las mediciones son simultáneas en ambos brazos para evitar el sesgo de las diferencias entre ambos brazos ${ }^{21}$, descritas aun en embarazos normales ${ }^{22}$. La modificación forzosa para este estudio fue el uso en todas las embarazadas del brazalete mediano (22-32 cm) único proporcionado con cada dispositivo, al margen de la circunferencia braquial, por lo cual ésta no se midió en este estudio; los de otros calibres para dichos dispositivos no están disponibles en el mercado local. 
Las lecturas de presión arterial se realizaron después de un periodo de cinco minutos de reposo en posición sedente y con los brazos de la embarazada al nivel del corazón. En la sede del estudio hay sillas ex profeso con descansabrazos ajustables en ambos lados y respaldo; se observaron las recomendaciones conocidas sobre las condiciones y posición para las lecturas ${ }^{11}$. Se realizó un mínimo de tres mediciones braquiales consecutivas en ambos brazos por intervalos de un minuto hasta que la diferencia entre presiones sistólicas fuera $\leq 10 \mathrm{mmHg}$ e $\leq 6 \mathrm{mmHg}$ en las diastólicas; este criterio define el estado basal de la embarazada. Los dispositivos electrónicos automáticos fueron del mismo modelo durante todo el estudio y cumplieron con el requisito de calibración. Se utilizaron dos dispositivos idénticos por cada paciente para medir simultáneamente en ambos brazos. La PAM para cada medición y en cada brazo se calculó con base a la fórmula conocida PD + (PS-PD)/3. La PAM de estudio resulta del promedio de las últimas dos mediciones estables $^{16}$. En este trabajo se agregaron las presiones sistólica (PS) y diastólica (PD) para estudio.

Los dispositivos electrónicos automáticos empleados en este estudio fueron marca Omron® (Japón), modelo HEM-7121 que cumple con el requisito $510(k)$ de la FDA.

El personal médico, paramédico y de enfermería encargado de las lecturas recibió capacitación para el uso de los dispositivos electrónicos, sobre el protocolo estandarizado, y se supervisó durante el estudio.

\section{Análisis estadístico}

Se aplicó estadística descriptiva para las distribuciones globales de las PS, PD y PAM en el embarazo y generar las ecuaciones generales. Los intervalos de referencia por semanas de gestación para las presiones arteriales PS, PD y PAM se elaboraron de modo similar a lo publicado por otros autore ${ }^{10,18}$ siguiendo el método de Royston y Wright ${ }^{23}$. Los múltiplos de la mediana (MoM) corregidos por gestación y peso materno se calcularon de manera habitual ${ }^{24}$. Las curvas se ajustaron a ecuaciones polinómicas mediante regresión de los cuadrados-mínimos; el modelo se seleccionó con base en los índices de bondad de ajuste, el análisis de residuales, la inspección y la simplicidad.

Se evaluaron los efectos de las características maternas y del embarazo sobre la PA mediante regresión lineal múltiple; el análisis fue escalonado para detectar efectos de confusión e interacción. El mejor modelo para cada PA se seleccionó con base en los índices de rendimiento, la congruencia clínica y el análisis de residuales. El modelo para calcular el percentil multivariado se basa en el método de Royston y Wright $^{23}$ modificado: la mediana se obtuvo de todas las variables incluidas en el modelo final y no sólo por edad de gestación; se analizó la consistencia del modelo en cada variable independiente.

Los supuestos de normalidad que requirieron una corroboración formal se evaluaron con la prueba de Shapiro-Wilk ${ }^{25,26}$; con base en el resultado se aplicaron estadísticos paramétricos, no paramétricos o se transformaron las variables antes de su análisis. Para el análisis multivariado se corroboraron adicionalmente los supuestos de varianzas similares, relación lineal y colinealidad, al transformar la variable en caso necesario. El nivel de significancia fue $\alpha=0.05$ para las pruebas estadísticas del valor de $p$.

La base de datos se creó desde los programas Astraia $^{\circledR}$ (Alemania) y Excel $^{\circledR}$ (EE.UU.); DOI: 10.6084/m9. figshare.12264152. El análisis estadístico se realizó en los programas Excel $^{\circledR}$ (EE.UU.) y MATLAB ${ }^{\circledR}$ (EE.UU.), con sus debidas licencias.

\section{Resultados}

La muestra poblacional se integró entre enero de 2017 y julio de 2018; quedó conformada por 1,056 mujeres que cumplieron todos los criterios de inclusión. Se obtuvo un total de 1,915 mediciones entre 5 y 41 semanas de gestación; las características de la población estudiada se presentan en la Tabla 1 y no hubo población indígena.

En 416 embarazos (21.7\%) se requirieron cuatro o más lecturas para conseguir la definición operativa en este estudio del estado inicial de la embarazada.

La presión arterial global tuvo una distribución normal y se presenta en la Tabla 2. La fórmula para calcular el percentil crudo de la presión arterial en el embarazo sigue la ecuación de Gauss (p. ej., percentiles $5^{\circ}$ y $95^{\circ}$, Tabla 2 ).

La edad de gestación mostró una relación cuadrática en forma de "U" con las presiones arteriales, y fue menor en el segundo trimestre. Los nomogramas por semanas de gestación se representan en la Figura 1 y los intervalos de referencia se enlistan en la Tabla 3. El modelo para el percentil de cada presión arterial por edad de gestación y para los MoM corregidos por peso materno se presenta en la Tabla 4.

Para el análisis multivariado se excluyeron tres registros de etnia africana y una del sur asiático por número insuficiente. La relación entre PS y PD fue de tipo lineal 


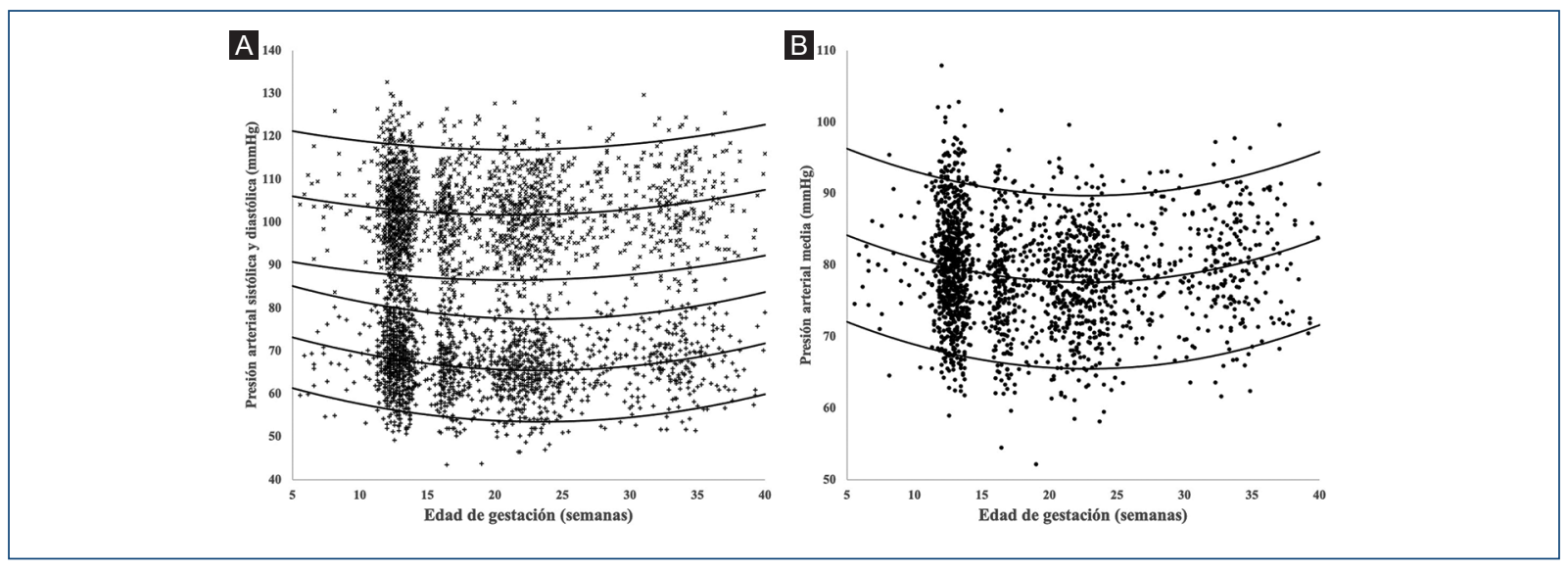

Figura 1. Diagrama de dispersión. A: Presión arterial sistólica $(x)$ con diastólica (+). B: Presión arterial media contra edad gestacional en esta población. Se muestran las medianas calculadas, con los respectivos percentiles $5^{\circ}$ y $95^{\circ}$.

Tabla 1. Características demográficas de la muestra poblacional

\begin{tabular}{|c|c|c|}
\hline Variable & \multicolumn{2}{|c|}{ Valor } \\
\hline $\begin{array}{l}\text { Edad materna en años, } \\
\text { mediana (IO) }\end{array}$ & 33.0 & $(29.0 ; 36.0)$ \\
\hline Peso materno en $\mathrm{kg}$, mediana (IQ) & 65.0 & $(58.4 ; 73)$ \\
\hline $\begin{array}{l}\text { Estatura materna en } \mathrm{cm} \text {, } \\
\text { mediana (IQ) }\end{array}$ & 160.0 & $(156 ; 164)$ \\
\hline $\begin{array}{l}\text { Índice de masa corporal, } \\
\text { mediana (IQ) }\end{array}$ & 25.3 & $(23 ; 28.1)$ \\
\hline $\begin{array}{l}\text { Etnia } \\
\text { Caucásico/hispano, n (\%) } \\
\text { Afrocaribeño, n (\%) } \\
\text { Sur asiático, n (\%) } \\
\text { Este asiático, n (\%) } \\
\text { Otro, mixto, n (\%) }\end{array}$ & $\begin{array}{c}1601 \\
3 \\
1 \\
24 \\
286\end{array}$ & $\begin{array}{c}(83.6 \%) \\
(0.2 \%) \\
(0.1 \%) \\
(1.3 \%) \\
(14.9 \%)\end{array}$ \\
\hline $\begin{array}{l}\text { Paridad } \\
\text { Nulípara, n (\%) } \\
\text { Paridad sin preeclampsia, n (\%) } \\
\text { Paridad con preeclampsia previa, } \\
\text { n }(\%)\end{array}$ & $\begin{array}{c}1,041 \\
843 \\
31\end{array}$ & $\begin{array}{c}(54.4 \%) \\
(44.0 \%) \\
(1.6 \%)\end{array}$ \\
\hline $\begin{array}{l}\text { Hábito de fumar } \\
\text { Fumadora, n (\%) } \\
\text { Dejó de fumar, n (\%) } \\
\text { No fuma, n }(\%)\end{array}$ & $\begin{array}{c}8 \\
224 \\
1,680\end{array}$ & $\begin{array}{c}(0.4 \%) \\
(11.7 \%) \\
(87.7 \%)\end{array}$ \\
\hline $\begin{array}{l}\text { Antecedente familiar de } \\
\text { preeclampsia, } \mathrm{n}(\%)\end{array}$ & 56 & $(2.9 \%)$ \\
\hline
\end{tabular}

con una correlación $r=0.71$ (Pearson, $p<0.001$ ), constante de 42.8 (IC95, 40.1, 45.5) y coeficiente de 0.89 (IC95, 0.85, 0.93). La correlación r entre PS y PD fue menor al valor crítico de 0.8 de colinealidad. Se desarrollaron modelos multivariados para las presiones PS, PD y PAM, incluida la PD en el modelo de la PS
Tabla 2. Presión arterial $(\mathrm{mmHg})$ general en el embarazo

\begin{tabular}{|l|c|c|c|}
\hline Variable & Valor & $\mathbf{p 5}$ & $\mathbf{p 9 5}$ \\
\hline $\begin{array}{l}\text { Presión arterial } \\
\text { sistólica, } \\
\text { promedio } \pm \text { DE }\end{array}$ & $102.7 \pm 9.30$ & 87.4 & 118.0 \\
\hline $\begin{array}{l}\text { Presión arterial } \\
\text { diastólica, } \\
\text { promedio } \pm \text { DE }\end{array}$ & $67.2 \pm 7.38$ & 55.1 & 79.3 \\
\hline $\begin{array}{l}\text { Presión arterial } \\
\text { media, } \\
\text { promedio } \pm \text { DE }\end{array}$ & $79.0 \pm 7.44$ & 66.8 & 91.3 \\
\hline
\end{tabular}

y viceversa; sólo la edad de gestación requirió transformación cuadrática, centrada en el valor crítico mínimo de cada presión arterial.

Los coeficientes de cada modelo final multivariado y sus estadísticos se presentan en las Tablas 5 a 7 para PS, PD y PAM, respectivamente. Los efectos de confusión se identificaron durante el proceso escalonado de cada modelo; se comentan a continuación (los coeficientes intermedios no se muestran).

La PD fue el principal factor determinante de la PS, al nulificar el efecto de la gestación en ésta y con efecto de confusión sobre las características maternas de peso, edad, estatura, etnia, paridad, dejar de fumar y antecedente familiar de preeclampsia. El peso materno, segundo determinante de la PS (valores T), tuvo efecto de confusión sobre la edad de gestación y las características maternas, excepto la estatura. En el modelo final quedaron excluidas la edad de gestación $(F=0.4566, p=0.499$ ), la etnia (otro, mixto $F=0.3798$, $p=0.538)$ y el este asiático $(F=0.8114, p=0.368)$. 
Tabla 3. Intervalos de referencia de la presión arterial por edad de gestación

\begin{tabular}{|c|c|c|c|c|c|c|c|c|c|}
\hline \multirow{2}{*}{$\begin{array}{l}\text { Semana de } \\
\text { gestación }\end{array}$} & \multicolumn{3}{|c|}{ PS } & \multicolumn{3}{|c|}{ PD } & \multicolumn{3}{|c|}{ PAM } \\
\hline & p5 & Mediana & p95 & p5 & Mediana & p95 & p5 & Mediana & p95 \\
\hline 5 & 90.9 & 106.1 & 121.3 & 61.4 & 73.3 & 85.2 & 72.1 & 84.2 & 96.3 \\
\hline 6 & 90.3 & 105.6 & 120.8 & 60.5 & 72.5 & 84.4 & 71.4 & 83.5 & 95.6 \\
\hline 7 & 89.9 & 105.1 & 120.3 & 59.8 & 71.7 & 83.6 & 70.7 & 82.8 & 94.9 \\
\hline 8 & 89.4 & 104.6 & 119.9 & 59.0 & 71.0 & 82.9 & 70.1 & 82.2 & 94.3 \\
\hline 9 & 89.0 & 104.2 & 119.4 & 58.3 & 70.3 & 82.2 & 69.5 & 81.6 & 93.7 \\
\hline 10 & 88.6 & 103.8 & 119.1 & 57.7 & 69.6 & 81.6 & 68.9 & 81.0 & 93.1 \\
\hline 11 & 88.3 & 103.5 & 118.7 & 57.1 & 69.1 & 81.0 & 68.4 & 80.5 & 92.6 \\
\hline 12 & 88.0 & 103.2 & 118.4 & 56.6 & 68.5 & 80.4 & 68.0 & 80.1 & 92.2 \\
\hline 13 & 87.7 & 102.9 & 118.1 & 56.1 & 68.0 & 79.9 & 67.5 & 79.6 & 91.7 \\
\hline 14 & 87.4 & 102.6 & 117.9 & 55.6 & 67.5 & 79.5 & 67.2 & 79.2 & 91.3 \\
\hline 15 & 87.2 & 102.4 & 117.6 & 55.2 & 67.1 & 79.1 & 66.8 & 78.9 & 91.0 \\
\hline 16 & 87.0 & 102.2 & 117.5 & 54.8 & 66.8 & 78.7 & 66.5 & 78.6 & 90.7 \\
\hline 17 & 86.9 & 102.1 & 117.3 & 54.5 & 66.5 & 78.4 & 66.2 & 78.3 & 90.4 \\
\hline 18 & 86.7 & 102.0 & 117.2 & 54.2 & 66.2 & 78.1 & 66.0 & 78.1 & 90.2 \\
\hline 19 & 86.7 & 101.9 & 117.1 & 54.0 & 66.0 & 77.9 & 65.8 & 77.9 & 90.0 \\
\hline 20 & 86.6 & 101.8 & 117.0 & 53.8 & 65.8 & 77.7 & 65.7 & 77.8 & 89.9 \\
\hline 21 & 86.6 & 101.8 & 117.0 & 53.7 & 65.7 & 77.6 & 65.6 & 77.7 & 89.8 \\
\hline 22 & 86.6 & 101.8 & 117.0 & 53.6 & 65.6 & 77.5 & 65.6 & 77.6 & 89.7 \\
\hline 23 & 86.6 & 101.8 & 117.1 & 53.6 & 65.5 & 77.5 & 65.5 & 77.6 & 89.7 \\
\hline 24 & 86.7 & 101.9 & 117.1 & 53.6 & 65.5 & 77.5 & 65.6 & 77.7 & 89.8 \\
\hline 25 & 86.8 & 102.0 & 117.2 & 53.6 & 65.6 & 77.5 & 65.6 & 77.7 & 89.8 \\
\hline 26 & 87.0 & 102.2 & 117.4 & 53.7 & 65.7 & 77.6 & 65.7 & 77.8 & 89.9 \\
\hline 27 & 87.1 & 102.3 & 117.6 & 53.9 & 65.8 & 77.8 & 65.9 & 78.0 & 90.1 \\
\hline 28 & 87.3 & 102.5 & 117.8 & 54.1 & 66.0 & 78.0 & 66.1 & 78.2 & 90.3 \\
\hline 29 & 87.6 & 102.8 & 118.0 & 54.3 & 66.3 & 78.2 & 66.3 & 78.4 & 90.5 \\
\hline 30 & 87.8 & 103.1 & 118.3 & 54.6 & 66.5 & 78.5 & 66.6 & 78.7 & 90.8 \\
\hline 31 & 88.1 & 103.4 & 118.6 & 54.9 & 66.9 & 78.8 & 66.9 & 79.0 & 91.1 \\
\hline 32 & 88.5 & 103.7 & 118.9 & 55.3 & 67.2 & 79.2 & 67.3 & 79.4 & 91.5 \\
\hline 33 & 88.8 & 104.1 & 119.3 & 55.7 & 67.7 & 79.6 & 67.7 & 79.8 & 91.9 \\
\hline 34 & 89.2 & 104.5 & 119.7 & 56.2 & 68.1 & 80.1 & 68.1 & 80.2 & 92.3 \\
\hline 35 & 89.7 & 104.9 & 120.1 & 56.7 & 68.6 & 80.6 & 68.6 & 80.7 & 92.8 \\
\hline 36 & 90.2 & 105.4 & 120.6 & 57.2 & 69.2 & 81.1 & 69.2 & 81.3 & 93.3 \\
\hline 37 & 90.7 & 105.9 & 121.1 & 57.9 & 69.8 & 81.7 & 69.7 & 81.8 & 93.9 \\
\hline 38 & 91.2 & 106.4 & 121.6 & 58.5 & 70.4 & 82.4 & 70.3 & 82.4 & 94.5 \\
\hline 39 & 91.7 & 107.0 & 122.2 & 59.2 & 71.1 & 83.1 & 71.0 & 83.1 & 95.2 \\
\hline 40 & 92.3 & 107.6 & 122.8 & 59.9 & 71.9 & 83.8 & 71.7 & 83.8 & 95.9 \\
\hline
\end{tabular}


Tabla 4. Modelo de la presión arterial por edad de gestación y corrección por peso

\begin{tabular}{|c|c|c|c|c|c|c|c|}
\hline Término & Cálculo & ES & valor T & valor $\mathbf{p}$ & RECM* & $r^{2}$ & $F$ vs. $K^{* *}$ \\
\hline $\begin{array}{l}\text { PS } \\
\text { Gestación } \\
\text { Constante } \\
\text { Semanas }^{\dagger} \\
\text { (Semanas)^2 }^{\wedge} \\
\text { Desviación estándar } \\
\text { MoM Peso } \\
\text { Constante } \\
\text { kg }\end{array}$ & $\begin{array}{c}109.12 \\
-0.6916 \\
0.01632 \\
9.2522 \\
\\
0.820 \\
0.002701\end{array}$ & $\begin{array}{l}1.7865 \\
0.1760 \\
0.0039\end{array}$ & $\begin{array}{l}61.1 \\
-3.9 \\
4.2\end{array}$ & $\begin{array}{l}<0.001 \\
<0.001 \\
<0.001 \\
\\
\quad<0.001 \\
<0.001\end{array}$ & 9.2571 & 0.0088 & 9.47 \\
\hline $\begin{array}{l}\text { PD } \\
\text { Gestación } \\
\text { Constante } \\
\text { Semanas } \\
\left(\text { Semanas) }{ }^{\wedge} 2\right. \\
\text { Desviación estándar } \\
\text { MoM Peso } \\
\text { Constante } \\
\text { kg }\end{array}$ & $\begin{array}{c}78.10758 \\
-1.07643 \\
0.023022 \\
7.2614 \\
\\
0.856 \\
0.00216\end{array}$ & $\begin{array}{l}1.4021 \\
0.1381 \\
0.0031 \\
\\
0.0143 \\
0.0002\end{array}$ & $\begin{array}{c}55.7 \\
-7.8 \\
7.5\end{array}$ & $\begin{array}{l}<0.001 \\
<0.001 \\
<0.001 \\
\\
<0.001 \\
<0.001\end{array}$ & 0.1052 & 0.0302 & 30.8 \\
\hline $\begin{array}{l}\text { PAM } \\
\text { Gestación } \\
\text { Constante } \\
\text { Semanas } \\
{\text { (Semanas) }{ }^{\wedge} 2} \\
\text { Desviación estándar } \\
\text { MoM Peso (recíproco) } \\
\text { Constante } \\
1 / \mathrm{kg} \\
(1 / \mathrm{kg})^{\wedge} 2\end{array}$ & $\begin{array}{c}88.45 \\
-0.9481 \\
0.02079 \\
7.3534 \\
\\
1.385 \\
-39.707 \\
932.096\end{array}$ & $\begin{array}{l}1.4198 \\
0.1399 \\
0.0031 \\
\\
\\
0.0557 \\
7.1454 \\
226.525\end{array}$ & $\begin{array}{c}62.3 \\
-6.8 \\
6.7\end{array}$ & $\begin{array}{l}<0.001 \\
<0.001 \\
<0.001 \\
\\
\quad<0.001 \\
<0.001 \\
<0.001\end{array}$ & 7.3572 & 0.0225 & 91.4 \\
\hline
\end{tabular}

La PS, principal factor determinante de la PD, tuvo efecto de confusión sobre todas las variables maternas y de gestación. La variable con el mayor efecto estadístico sobre la PAM fue el peso materno y no se le encontraron variables de confusión; por el contrario, el peso materno tuvo efecto de confusión para la edad materna, etnia, preeclampsia previa, fumar, dejar de fumar y antecedente familiar de preeclampsia. La edad de gestación fue la segunda en efecto estadístico sobre la PAM, independiente del peso materno, y tuvo efecto de confusión para edad materna, estatura y fumar. No hubo efecto de confusión ni de interacción entre peso materno y edad de gestación. La etnia tuvo efecto de confusión sobre la estatura materna y el antecedente familiar de preeclampsia sobre el grupo étnico diverso y mixto. Por su parte, la paridad fue variable de confusión para la edad materna, fumar y el antecedente familiar de preeclampsia. La única característica excluida del modelo final de la PAM fue el hábito de fumar ( $F=0.1741, p=0.677$ ).

Del análisis de residuales se determinó una desviación estándar constante en cada modelo para calcular el percentil personalizado de cada presión; se muestran en su respectiva tabla. Los modelos se han codificado en archivo, DOI: 10.6084/m9.figshare.12268328.

\section{Discusión}

Las distribuciones generales de PS, PD y MAP resultaron por debajo de los estándares convencionales con o sin embarazo ${ }^{1-4,11}$, similar a lo descrito en mujeres no gestantes de una población diferente a ésta ${ }^{27}$. Nótese que el umbral de "presión arterial elevada" según el $\mathrm{ACC}^{6}, 120 / 80 \mathrm{mmHg}$, equivale al percentil $97^{\circ}$ tanto en esta población de embarazadas como en las mujeres no gestantes del estudio referido ${ }^{27}$; además, se ha demostrado que este umbral de 120/80 mmHg tiene valor en la predicción de preeclampsia y otros desenlaces perinatales adversos en otros estudios ${ }^{7,8}$. Por lo tanto, se deben revisar los criterios diagnósticos de hipertensión en el embarazo. Este estudio propone una definición estadística de normalidad; sin embargo, se requieren otros longitudinales para responder de forma satisfactoria este aspecto. 
Tabla 5. Modelo multivariado de la presión arterial sistólica en el embarazo

\begin{tabular}{|c|c|c|c|c|}
\hline Término & Cálculo & ES & valor $\mathbf{T}$ & valor $p$ \\
\hline $\begin{array}{l}\text { Valor medio esperado } \\
\text { Intersección } \\
\text { Presión diastólica }\end{array}$ & $\begin{array}{l}18.7195 \\
1.1901\end{array}$ & $\begin{array}{l}9.3639 \\
0.1136\end{array}$ & $\begin{array}{c}2.0 \\
10.5\end{array}$ & $\begin{array}{l}0.046 \\
<0.001\end{array}$ \\
\hline $\begin{array}{l}\text { Peso materno } \\
\text { Presión diastólica:peso }\end{array}$ & $\begin{array}{l}0.5418 \\
-0.0054\end{array}$ & $\begin{array}{l}0.1182 \\
0.0017\end{array}$ & $\begin{array}{c}4.6 \\
-3.2\end{array}$ & $\begin{array}{c}<0.001 \\
0.001\end{array}$ \\
\hline Edad materna & 0.0359 & 0.0314 & 1.1 & 0.252 \\
\hline Estatura materna & -0.0591 & 0.0359 & -1.6 & 0.100 \\
\hline $\begin{array}{l}\text { Paridad }^{\dagger} \\
\text { Preeclampsia previa } \\
\text { Edad:preeclampsia previa } \\
\text { Nulípara } \\
\text { Estatura:nulípara }\end{array}$ & $\begin{array}{c}20.1042 \\
-0.5302 \\
-15.8734 \\
0.1028\end{array}$ & $\begin{array}{l}7.8066 \\
0.2261 \\
7.3952 \\
0.0461\end{array}$ & $\begin{array}{c}2.6 \\
-2.3 \\
-2.1 \\
2.2\end{array}$ & $\begin{array}{l}0.010 \\
0.019 \\
0.032 \\
0.026\end{array}$ \\
\hline $\begin{array}{l}\text { Fuma } \\
\text { Peso:fuma }\end{array}$ & $\begin{array}{c}-35.6249 \\
0.5711\end{array}$ & $\begin{array}{c}14.2488 \\
0.2283\end{array}$ & $\begin{array}{l}-2.5 \\
2.5\end{array}$ & $\begin{array}{l}0.012 \\
0.012\end{array}$ \\
\hline $\begin{array}{l}\text { Dejó de fumar } \\
\text { Peso:dejó de fumar }\end{array}$ & $\begin{array}{l}6.2387 \\
-0.0937\end{array}$ & $\begin{array}{l}2.7398 \\
0.0404\end{array}$ & $\begin{array}{c}2.3 \\
-2.3\end{array}$ & $\begin{array}{l}0.023 \\
0.020\end{array}$ \\
\hline Antecedente familiar de preeclampsia & 1.4381 & 0.8565 & 1.7 & 0.093 \\
\hline Desviación estándar & 6.2315 & & & \\
\hline
\end{tabular}

*Raíz del error cuadrático medio $=6.254$.

R-cuadrado: 0.551, ajustado: 0.548 .

Estadístico-Fvs. Modelo constante: 166 , valor $p<0.001$

${ }^{\dagger} \mathrm{Grupo}$ de referencia: caucásico/hispano con paridad sin preeclampsia.

La distribución MoM fue $1.000 \pm 0.061 \mathrm{DE}$ (Shapiro-Wilk $=0.9975, \mathrm{p}=0.004$ ).

El puntaje $Z$ tuvo distribución $0 \pm 1.0 \mathrm{DE}$ (Shapiro-Wilk $=0.9981, p=0.024)$.

No está previsto responder en este estudio la utilización de sólo tres lecturas o las que sean necesarias; sin embargo es llamativo que alrededor de una quinta parte de los casos necesitó cuatro o más lecturas para conseguir la definición operativa, aquí establecida para el estado basal de la embarazada. La significancia de este hallazgo puede ser motivo de otro estudio.

El sesgo esperado en los valores de la presión arterial por el uso forzoso del brazalete único ${ }^{11}$ no afecta la validez de los modelos porque el ajuste por peso materno podría corregir el efecto de la circunferencia braquial ${ }^{28}$. Esta hipótesis debe ser objeto de otro estudio.

La relación entre la PS y la PD con la edad de gestación se expresa aquí mediante ecuaciones polinómicas, similar a lo hecho en un metaanálisis ${ }^{10}$. Las curvas para PD resultaron casi idénticas entre ambos estudios, no así las de presión PS. Esto podría deberse a comportamiento consistente a lo largo de la gestación humana para la PD, mas no para la PS, según lo explican los modelos multivariados, en los que la edad de gestación sólo mantiene efecto significativo para la presión PD. Aunque la PS puede expresarse por gestación, esta relación pierde significancia frente a las características maternas, sus principales determinantes; este hallazgo es congruente con otro estudio, en el cual se cuantificó el volumen sistólico a lo largo de la gestación, pero también se determinó en particular por características maternas ${ }^{29}$. Por lo tanto, los cambios fisiológicos del embarazo como el volumen circulante, la viscosidad sanguínea, el ritmo cardíaco y las resistencias periféricas se reflejan sobre todo en la PD, la cual a su vez explica aproximadamente $50 \%$ de la variación en la PS (esta cuantificación es única en las publicaciones médicas). Al corregirse este efecto, la PS refleja entonces el estado cardiovascular con el que se embaraza la mujer, pero no de origen poblacional o étnico, sino del historial médico: ginecoobstétrico y tabaco.

La relación cuadrática aquí descrita entre la PAM y la gestación es similar a lo publicado por el grupo que diseñó el método estandarizado original ${ }^{18}$ : fue menor en el segundo trimestre con varianza constante a lo largo de la gestación. Es posible estandarizar la medición de la presión arterial a lo largo del embarazo.

Similar a las PS y PD, las características maternas, sobre todo el peso materno, tuvieron efecto 
Tabla 6. Modelo multivariado de la presión arterial diastólica en el embarazo

\begin{tabular}{|c|c|c|c|c|}
\hline Término & Cálculo & ES & $\begin{array}{l}\text { valor } \\
T\end{array}$ & $\begin{array}{c}\text { valor } \\
\mathbf{p}\end{array}$ \\
\hline $\begin{array}{l}\text { Valor medio esperado } \\
\text { Intersección } \\
\text { Presión sistólica }\end{array}$ & $\begin{array}{l}-82.3298 \\
1.5106\end{array}$ & $\begin{array}{c}33.2113 \\
0.3217\end{array}$ & $\begin{array}{l}-2.5 \\
4.7\end{array}$ & $\begin{array}{c}0.013 \\
<0.001\end{array}$ \\
\hline $\begin{array}{l}\text { Estatura materna } \\
\text { Presión } \\
\text { sistólica:estatura }\end{array}$ & $\begin{array}{c}0.5902 \\
-0.0061\end{array}$ & $\begin{array}{l}0.2068 \\
0.0020\end{array}$ & $\begin{array}{l}2.9 \\
-3.0\end{array}$ & $\begin{array}{l}0.004 \\
0.003\end{array}$ \\
\hline $\begin{array}{l}\text { Gestación }^{\dagger} \\
\text { Estatura:gestación }\end{array}$ & $\begin{array}{l}-0.0911 \\
0.0007\end{array}$ & $\begin{array}{l}0.0509 \\
0.0003\end{array}$ & $\begin{array}{c}-1.8 \\
2.2\end{array}$ & $\begin{array}{l}0.074 \\
0.027\end{array}$ \\
\hline Edad materna & -0.0614 & 0.0391 & -1.6 & 0.116 \\
\hline Peso materno & -0.000003 & 0.0121 & 0.0 & 1.000 \\
\hline $\begin{array}{l}\text { Fuma } \\
\text { Peso:fuma }\end{array}$ & $\begin{array}{l}31.2338 \\
-0.4958\end{array}$ & $\begin{array}{c}11.5455 \\
0.1850\end{array}$ & $\begin{array}{l}2.7 \\
-2.7\end{array}$ & $\begin{array}{l}0.007 \\
0.007\end{array}$ \\
\hline $\begin{array}{l}\text { Dejó de fumar } \\
\text { Presión } \\
\text { sistólica:dejó de } \\
\text { fumar }\end{array}$ & $\begin{array}{l}-9.6010 \\
0.0889\end{array}$ & $\begin{array}{l}4.0611 \\
0.0395\end{array}$ & $\begin{array}{c}-2.4 \\
2.2\end{array}$ & $\begin{array}{l}0.018 \\
0.025\end{array}$ \\
\hline $\begin{array}{l}\text { Paridad }^{\ddagger} \\
\text { Preeclampsia previa } \\
\text { Peso:preeclampsia } \\
\text { previa } \\
\text { Nulípara } \\
\text { Estatura:nulípara } \\
\text { Gestación:nulípara } \\
\text { Edad:nulípara }\end{array}$ & $\begin{array}{c}14.4581 \\
-0.1799 \\
12.9214 \\
-0.1013 \\
-0.0105 \\
0.1248\end{array}$ & $\begin{array}{l}5.4690 \\
0.0725 \\
6.2079 \\
0.0375 \\
0.0041 \\
0.0513\end{array}$ & $\begin{array}{c}2.6 \\
-2.5 \\
2.1 \\
-2.7 \\
-2.5 \\
2.4\end{array}$ & $\begin{array}{l}0.008 \\
0.013 \\
0.038 \\
0.007 \\
0.012 \\
0.015\end{array}$ \\
\hline $\begin{array}{l}\text { Etnia }^{\ddagger} \\
\text { Otro, mixto. } \\
\text { Estatura:otro, } \\
\text { mixto } \\
\text { Nulípara:otro, } \\
\text { mixto } \\
\text { Este asiático } \\
\text { Presión } \\
\text { sistólica:este } \\
\text { asiático }\end{array}$ & $\begin{array}{c}-19.1500 \\
0.1161 \\
1.5075 \\
-25.1961 \\
0.2729\end{array}$ & $\begin{array}{c}8.0731 \\
0.0508 \\
0.6625 \\
12.1923 \\
0.1205\end{array}$ & $\begin{array}{c}-2.4 \\
2.3 \\
2.3 \\
-2.1 \\
2.3\end{array}$ & $\begin{array}{l}0.018 \\
0.022 \\
0.023 \\
0.039 \\
0.024\end{array}$ \\
\hline Desviación estándar & 5.0355 & & & \\
\hline
\end{tabular}

*Raíz del error cuadrático medio $=5.065$.

R-cuadrado: 0.533, ajustado: 0.528 .

Estadístico-F vs modelo constante: 98.1 valor $\mathrm{p}<0.001$

†Edad de gestación en semanas, centrada en 23s 3d y con transformación cuadrática.

Gestación $=((\text { semanas }+ \text { días/7) })-(23+3 / 7))^{\wedge} 2$.

${ }^{\ddagger}$ Grupo de referencia: caucásico/hispano con paridad sin preeclampsia.

La distribución MoM fue $1.000 \pm 0.075 \mathrm{DE}$ (Shapiro-Wilk $=0.9990, \mathrm{p}=0.356$ ).

El puntaje $Z$ tuvo distribución $0 \pm 1.0 \mathrm{DE}$ (Shapiro-Wilk $=0.9985, \mathrm{p}=0.081$ ).

determinante sobre la PAM, no secundario o de corrección a la gestación, como sería esperable ${ }^{12,24}$. Aunque los intervalos de referencia de la presión arterial se construyen de acuerdo con la gestación ${ }^{10,18}$, deben corregirse por características maternas.
Los efectos de las características maternas sobre la PAM que han descrito otros especialistas ${ }^{12}$ tienen similitudes con estos hallazgos; impresionan las diferencias de origen poblacional.

Por diseño no es posible diferenciar entre el efecto fisiológico y el predictivo para preeclampsia previa ${ }^{12}$, antecedente familiar de preeclampsia ${ }^{12,15}$ y dejar de fumar ${ }^{30}$. La aplicación de los modelos presentados aquí debe ser transversal (diagnóstica).

Las investigaciones futuras precisarán lo anterior y otros aspectos, entre ellos el efecto del momento en que se dejó de fumar ${ }^{31}$, el alcance de su aplicación y la utilidad en otros grupos como diabéticas e hipertensas y la relación de COVID-19 con preeclampsia ${ }^{32}$.

\section{Conclusiones}

Es necesario revisar los criterios diagnósticos para hipertensión en el embarazo. Es factible crear intervalos de referencia estandarizados de la presión arterial a lo largo de la gestación. La personalización por características maternas mejora la precisión del modelo, ya que son los principales determinantes de la presión arterial de la embarazada. La PS podría representar en particular el estado cardiovascular con el que se embaraza la mujer, mientras que la PD podría reflejar de manera primordial la adaptación materna a la gestación.

\section{Financiamiento}

Ninguno.

\section{Conflicto de intereses}

Los autores declaran no tener conflicto de intereses.

\section{Responsabilidades éticas}

Protección de personas y animales. Los autores declaran que para esta investigación no se han realizado experimentos en seres humanos ni en animales.

Confidencialidad de los datos. Los autores declaran que han seguido los protocolos de su centro de trabajo sobre la publicación de datos de pacientes. 
Tabla 7. Modelo multivariado de la presión arterial media en el embarazo

\begin{tabular}{|c|c|c|c|c|}
\hline Término & Cálculo & ES & valor $\mathbf{T}$ & valor $p$ \\
\hline $\begin{array}{l}\text { Valor medio esperado } \\
\text { Intersección } \\
\text { Peso materno }\end{array}$ & $\begin{array}{c}68.3306 \\
0.1898\end{array}$ & $\begin{array}{l}4.5517 \\
0.0155\end{array}$ & $\begin{array}{l}15.0 \\
12.3\end{array}$ & $\begin{array}{l}<0.001 \\
<0.001\end{array}$ \\
\hline Gestación $^{\dagger}$ & 0.0319 & 0.0044 & 7.2 & $<0.001$ \\
\hline Edad materna & -0.0495 & 0.0533 & -0.9 & 0.353 \\
\hline Estatura materna & -0.0176 & 0.0279 & -0.6 & 0.527 \\
\hline $\begin{array}{l}\text { Paridad }{ }^{\ddagger} \\
\text { Preeclampsia previa } \\
\text { Estatura:preeclampsia previa } \\
\text { Nulípara } \\
\text { Gestación:nulípara } \\
\text { Edad:nulípara }\end{array}$ & $\begin{array}{l}62.3303 \\
-0.3593 \\
-4.5627 \\
-0.0185 \\
0.2047\end{array}$ & $\begin{array}{r}28.8721 \\
0.1803 \\
2.3367 \\
0.0058 \\
0.0699\end{array}$ & $\begin{array}{l}2.2 \\
-2.0 \\
-2.0 \\
-3.2 \\
2.9\end{array}$ & $\begin{array}{l}0.031 \\
0.046 \\
0.051 \\
0.002 \\
0.003\end{array}$ \\
\hline $\begin{array}{l}\text { Etnia }^{\ddagger} \\
\text { Este asiático } \\
\text { Gestación:este asiático } \\
\text { Otro, mixto } \\
\text { Nulípara:otro, mixto }\end{array}$ & $\begin{array}{c}-1.8627 \\
0.0669 \\
-1.4160 \\
2.3129\end{array}$ & $\begin{array}{l}2.4780 \\
0.0308 \\
0.6598 \\
0.8998\end{array}$ & $\begin{array}{c}-0.8 \\
2.2 \\
-2.1 \\
2.6\end{array}$ & $\begin{array}{l}0.452 \\
0.030 \\
0.032 \\
0.010\end{array}$ \\
\hline Dejó de fumar & -0.8939 & 0.4967 & -1.8 & 0.072 \\
\hline Antecedente familiar de preeclampsia & 1.6792 & 0.9484 & 1.8 & 0.077 \\
\hline Desviación estándar & 6.9064 & & & \\
\hline
\end{tabular}

${ }^{*}$ Raíz del error cuadrático medio $=6.934$.

R-cuadrado: 0.139, ajustado: 0.133 .

Estadístico-F vs. modelo constante: 20.5 , valor $p<0.001$

${ }^{\dagger}$ Edad de gestación en semanas, centrada en 22s $6 \mathrm{~d}$ y con transformación cuadrática. Gestación $=\left((\text { semanas }+ \text { días/7) }-(22+6 / 7))^{\wedge} 2\right.$

${ }^{\ddagger}$ Grupo de referencia: caucásico/hispano con paridad sin preeclampsia.

La distribución MoM fue $1.000 \pm 0.087 \mathrm{DE}$ (Shapiro-Wilk $=0.9985, \mathrm{p}=0.091$ ).

El puntaje $Z$ tuvo distribución $0 \pm 1.0 \mathrm{DE}$ (Shapiro-Wilk $=0.9985, \mathrm{p}=0.091$ ).

Derecho a la privacidad y consentimiento informado. Los autores declaran que en este artículo no aparecen datos de pacientes.

\section{Bibliografía}

1. Tranquilli AL, Dekker G, Magee L, Roberts J, Sibai BM, Steyn W, et al. The classification, diagnosis and management of the hypertensive disorders of pregnancy: a revised statement from the ISSHP. Pregnancy Hypertens. 2014;4:97-104.

2. Lowe SA, Bowyer L, Lust K, McMahon LP, Morton M, North RA, et al. SOMANZ guidelines for the management of hypertensive disorders of pregnancy 2014. Aust NZJ Obstet Gynaecol. 2015;55:e1-e29.

3. ACOG Practice Bulletin No. 202: Gestational hypertension and preeclampsia. Obstet Gynecol. 2019;133:e1-e25.

4. Webster K, Fishburn S, Maresh M, Findlay SC, Chappell LC. Diagnosis and management of hypertension in pregnancy: summary of updated NICE guidance. BMJ. 2019;366:I5119.

5. Hypertension in pregnancy. Int J Gynecol Obstet. 1996;53:175-83.

6. Whelton PK, Carey RM, Aronow WS, Casey DE, Collins KJ, Dennison Himmelfarb C, et al. 2017 ACC/AHA/AAPA/ABC/ACPM/AGS/APhA/ ASH/ASPC/NMA/PCNA Guideline for the prevention, detection, evaluation, and management of high blood pressure in adults: a report of the American College of Cardiology/American Heart Association Task Force on Clinical Practice Guidelines. J Am Coll Cardiol. 2018;71:e127-e248.

7. Sutton EF, Hauspurg A, Caritis SN, Powers RW, Catov JM. Maternal outcomes associated with lower range stage 1 hypertension. Obstet Gynecol. 2018;132:843-9.
8. Reddy M, Rolnik DL, Harris K, Li W, Mol BW, Da Silva Costa F, et al. Challenging the definition of hypertension in pregnancy: a retrospective cohort study. Am J Obstet Gynecol. 2020;222:606.e1-.e21.

9. Wilson M, Morganti AA, Zervoudakis I, Letcher RL, Romney BM, Von Oeyon $\mathrm{P}$, et al. Blood pressure, the renin-aldosterone system and sex steroids throughout normal pregnancy. Am J Med. 1980;68:97-104.

10. Loerup L, Pullon RM, Birks J, Fleming S, Mackillop LH, Gerry S, et al. Trends of blood pressure and heart rate in normal pregnancies: a systematic review and meta-analysis. BMC Med. 2019;17:167.

11. Unger T, Borghi $C$, Charchar F, Khan NA, Poulter NR, Prabhakaran D, et al. 2020 International Society of Hypertension Global Hypertension Practice Guidelines. Hypertension. 2020;75:1334-57.

12. Wright A, Wright D, Ispas CA, Poon LC, Nicolaides KH. Mean arterial pressure in the three trimesters of pregnancy: effects of maternal characteristics and medical history. Ultrasound Obstet Gynecol. 2015;45:698-706.

13. Iwama $\mathrm{N}$, Metoki $\mathrm{H}$, Nishigori $\mathrm{H}$, Mizuno S, Takahashi $\mathrm{F}$, Tanaka $\mathrm{K}$ et al. Blood pressure changes during twin pregnancies: the Japan Environment and Children's Study. J Hypertens. 2019;37:206-15.

14. Kuleva M, Youssef A, Maroni E, Contro E, Pilu G, Rizzo N, et al. Maternal cardiac function in normal twin pregnancy: a longitudinal study. UItrasound Obstet Gynecol. 2011;38:575-80.

15. Wright D, Syngelaki A, Akolekar R, Poon LC, Nicolaides KH. Competing risks model in screening for preeclampsia by maternal characteristics and medical history. Am J Obstet Gynecol. 2015;213:62 e1-e10.

16. Poon LCY, Zymeri NA, Zamprakou A, Syngelaki A, Nicolaides KH. Protocol for measurement of mean arterial pressure at 11-13 weeks' gestation. Fetal Diagn Ther. 2012;31:42-8.

17. Tayyar A, Krithinakis K, Wright A, Wright D, Nicolaides KH. Mean arterial pressure at 12, 22, 32 and 36 weeks' gestation in screening for pre-eclampsia. Ultrasound Obstet Gynecol. 2016;47:573-9.

18. Roberts L, Chaemsaithong P, Sahota DS, Nicolaides KH, Poon LCY. Protocol for measurement of mean arterial pressure at 10-40weeks' gestation. Pregnancy Hypertens. 2017;10:155-60. 
Arch Cardiol Mex. 2021;91(3)

19. Poon LC, Shennan A, Hyett JA, Kapur A, Hadar E, Divakar H, et al. The International Federation of Gynecology and Obstetrics (FIGO) initiative on pre-eclampsia: a pragmatic guide for first-trimester screening and prevention. Int J Gynecol Obstet. 2019:145:1-33.

20. Oviedo-Cruz H R-MM, Cortés-Martínez MA. Intercambiabilidad entre e método por auscultación y por dispositivo electrónico automático de la presión arterial media en mujeres embarazadas sin hipertensión. Estudio piloto. Ginecol Obstet Mex. 2017:85:653-8.

21. Lane D, Beevers M, Barnes N, Bourne J, John A, Malins S, et al. Inter-arm differences in blood pressure: when are they clinically significant? J Hypertens. 2002;20:1089-95.

22. Poon L, Kametas N, Strobl I, Pachoumi C, Nicolaides K. Inter-arm blood pressure differences in pregnant women. BJOG. 2008:115:1122-30

23. Royston $P$, Wright EM. How to construct 'normal ranges' for fetal variables. Ultrasound Obstet Gynecol. 1998;11:30-8.

24. Reynolds TM, Vranken G, Van Nueten J. Weight correction of MoM values: which method? J Clin Pathol. 2006:59:753-8.

25. Altman DG, Bland JM. Statistics notes: the normal distribution. BMJ. 1995;310:298.
26. Ghasemi A, Zahedias। S. Normality tests for statistical analysis: a guide for non-statisticians. Int J Endocrinol Metab. 2012;10:486-9.

27. Villarreal-García E, Villarreal-García LV. Presión arterial en población indígena tzotzil de Chiapas, México. Arch Cardiol Mex. 2018:88:381-5.

28. Ng CM, Badon SE, Dhivyalosini M, Hamid JJM, Rohana AJ, Teoh AN, et al Associations of pre-pregnancy body mass index, middle-upper arm circumference, and gestational weight gain. Sex Reprod Healthc. 2019;20:60-5.

29. Vinayagam D, Thilaganathan B, Stirrup O, Mantovani E, Khalil A. Maternal hemodynamics in normal pregnancy: reference ranges and role of maternal characteristics. Ultrasound Obstet Gynecol. 2018;51:665-71.

30. Pipkin FB. Genetics of preeclampsia C. Smoking in moderate/severe preeclampsia worsens pregnancy outcome, but smoking cessation limits the damage. Hypertension. 2008;51:1042-6.

31. Wang X, Lee NL, Burstyn I. Maternal smoking and gestational hypertension: Heterogeneous effect by timing of the exposure. Pregnancy Hypertens. 2019;15:123-9.

32. Di Mascio D, Khalil A, Saccone G, Rizzo G, Buca D, Liberati M, et al. Outcome of coronavirus spectrum infections (SARS, MERS, COVID 1 -19) during pregnancy: a systematic review and meta-analysis. Am J Obstet Gynecol MFM. 2020;100107. 